\title{
Multiple endocrine neoplasia type 2 syndromes may be associated with renal malformations
}

\author{
F. LORÉ ${ }^{1}$, F. TALIDIS ${ }^{1}$, G. DI CAIRANO ${ }^{1} \&$ A. RENIERI ${ }^{2}$ \\ From the ${ }^{1}$ Endocrinology Unit; ${ }^{2}$ Medical Genetics Unit, University of Siena, Italy
}

\begin{abstract}
Loré F, Talidis F, Di Cairano G, Renieri A (Endocrinology Unit; Medical Genetics Unit, University of Siena, Italy). Multiple endocrine neoplasia type 2 syndromes may be associated with renal malformations. J Intern Med 2001; 250: 37-42.
\end{abstract}

Objective. The RET proto-oncogene is known to be the susceptibility gene for various disease phenotypes, including multiple endocrine neoplasia type 2 (MEN 2). Recent studies have also suggested an involvement of RET in the development of the mammalian kidney. Although kidney agenesis or dysgenesis has been observed in mice lacking functional ret, no clinically relevant kidney abnormalities have been reported in individuals with known RET mutations and familial medullary thyroid carcinoma (FMTC). We have studied a family with five members affected with isolated FMTC. DNA analysis was performed and the involved RET mutation was identified. Amongst these patients were a woman and her son.

Design. Case report.

Setting. University department.
Patients. A 32-year-old woman and her son with FMTC and unilateral renal agenesis.

Results. The woman's abdominal ultrasound findings demonstrated unilateral renal absence of the left kidney. Her son, when only a few months old, had undergone surgical treatment for Hirschsprung's disease. Abdominal ultrasonography was performed recently, and left-side renal absence was diagnosed. Intravenous pyelography confirmed the agenesis of his left kidney, whilst the contralateral kidney displayed compensatory hypertrophy.

Conclusions. The involvement of the RET protooncogene in the early growth and differentiation of the human kidney is now generally accepted. We believe that at least a proportion of patients with MEN 2 may have undiagnosed renal malformations. We suggest therefore that noninvasive imaging techniques, such as ultrasonography, should be used to explore the presence of renal abnormalities in subjects with demonstrated RET mutations.

Keywords: FMTC, germline mutations, Hirschsprung disease, kidney, MEN 2, RET proto-oncogene, thyroid neoplasms, urogenital abnormalities.

\section{Introduction}

Medullary thyroid carcinoma (MTC), a tumour of the parafollicular $\mathrm{C}$ cells of the thyroid, may occur in two presentations: a sporadic form, which accounts for the majority of cases, and a familial counterpart arising as the major component of an autosomal dominantly inherited cancer syndrome, multiple endocrine neoplasia type 2 (MEN 2) [1].

Depending on the tissues involved, MEN 2 is divided into three variants: MEN 2A (involving families with MTC, pheochromocytoma and, less commonly, hyperparathyroidism), MEN 2B (invol- ving families with MTC, with or without pheochromocytoma and with characteristic clinical abnormalities, such as ganglioneuromas of the lips, tongue and colon, but without hyperparathyroidism) and familial medullary thyroid carcinoma (FMTC).

Familial medullary thyroid carcinoma is clinically defined by the presence of MTC in a minimum of four family members, with no objective evidence of pheochromocytoma or parathyroid involvement on screening of all living affected and at-risk subjects $[2,3]$.

Germline mutations in the RET proto-oncogene, which encodes a cell-surface glycoprotein related to 
the family of receptor tyrosine kinases [4], have been identified in over $90 \%$ of families with MEN 2 [5].

Mutations of the RET gene are also the underlying genetic cause in a proportion of patients with Hirschsprung's disease, a congenital absence of innervation in the lower intestinal tract. In a very small fraction of kindreds, MEN 2 cosegregates with Hirschsprung's disease [6].

Recent studies have suggested that the RET protooncogene may also play an essential role in renal morphogenesis. It has been demonstrated that ret knockout mice (ret-l-) display kidney agenesis or severe kidney dysgenesis [7]. Moreover, one of the ligands for RET, the glial cell line-derived neurotrophic factor (GDNF), has also been shown to be essential for kidney development [8, 9]. However, no renal abnormalities have been reported amongst patients with known RET mutations in association with MTC.

We describe a family with FMTC and Hirschsprung's disease, in association with a known RET mutation, in which two members exhibit unilateral kidney agenesis [10].

\section{Case report}

The pedigree of the family is shown in Fig. 1. A 56-year-old woman (patient III-1), in whom MTC had been diagnosed at the age of 42 years, following a total thyroidectomy performed because of a suspect thyroid nodule, was referred to the Endocrinology Unit of our hospital for evaluation of a neck mass. She was shown to have high serum calcitonin levels and the diagnosis of lymph node metastasis of MTC was confirmed histologically.

Almost simultaneously, the 44-year-old brother of patient III-1 (patient III-2) underwent thyroidectomy in the surgical department of the same hospital and was diagnosed as having MTC. Following surgery, he was also referred to our department.

Elevated serum calcitonin levels (in the basal state and following pentagastrin stimulation) and subsequently available DNA analysis led to the identification of an additional family member with MTC (IV-1). Two other subjects at risk (V-2V-4), were demonstrated to have the same RET protooncogene mutation (C620S) as identified in the affected members of the family. The results of the genetic studies have been reported in a separate paper [11].

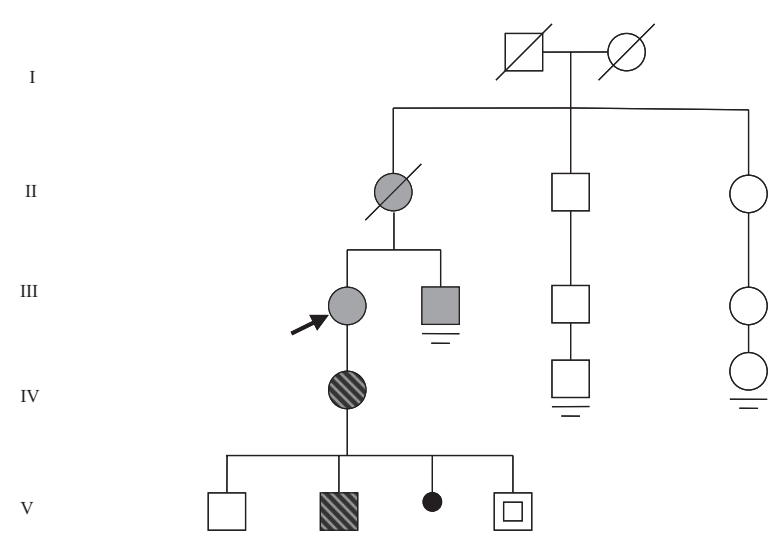

Fig. 1 Pedigree of the family. The arrow indicates the patient who brought the family to our attention (proband). Gray symbols indicate members with medullary thyroid carcinoma (MTC), hatched symbols the members with MTC and renal agenesis, and symbols with a slash deceased family members. The symbol with a square indicates a member with the C620S RET mutation, but with no evidence of thyroid tumour at the age of eight. The small blackened circle represents a miscarriage. The parallel bars indicate no offspring.

Subsequent research into the medical history of the parents of patients III- 1 and III- 2 revealed that the mother (patient II-1) had undergone total thyroidectomy in 1971 for a thyroid tumour which, at that time, had been histologically diagnosed as 'undifferentiated small cell carcinoma'. Recent histological re-examination of the surgical specimen using calcitonin antibodies led to the diagnosis of MTC 26 years after surgery.

Altogether, 13 family members were studied. The C620S RET mutation was detected in five of them, including subject $\mathrm{V}-4$, who, at the age of 8 years, had no evidence of MTC.

Medullary thyroid carcinoma was diagnosed in five patients, including patient II-1, who died of unknown causes in 1979, at the age of 63. PCR could not be performed on the tissue sections of this patient. Screening for MEN 2-associated tumours included 24-h urinary free catecholamines, urinary vanillylmandelic acid and serum calcium and parathyroid hormone levels in all the living affected and at-risk subjects.

\section{Patient IV-1}

A 32-year-old woman, the daughter of patient III-1, was one of the relatives contacted during our investigation. Serum calcitonin levels were found 
to be abnormally high, both in the basal state and following pentagastrin stimulation. Ultrasonography revealed two small hypoechoic nodules located in the right and left thyroid lobes (with a maximum diameter of 9 and $6 \mathrm{~mm}$, respectively). Fine needle aspiration biopsy revealed cytological findings sug- gesting thyroid cancer. The patient underwent total thyroidectomy with regional lymph node dissection. Histopathologic examination confirmed the diagnosis of MTC.

Abdominal ultrasonography demonstrated unilateral renal absence involving the left kidney. The
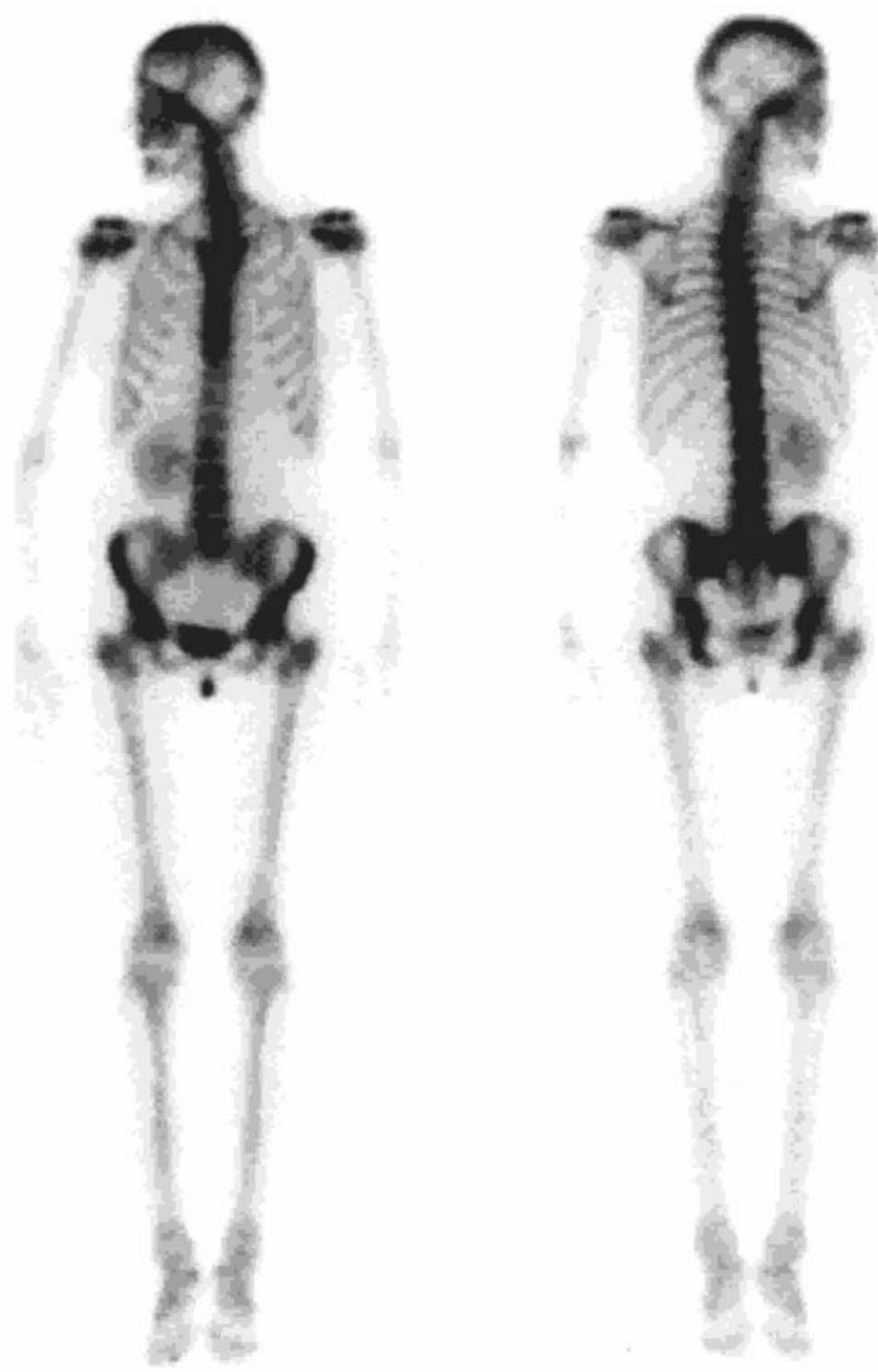

Fig. 2 Radionuclide imaging ( ${ }^{99} \mathrm{~m}$ Tc methylene bisphosphonate) of patient IV-1: absence of the left kidney and hypertrophic right kidney. (C) 2001 Blackwell Science Ltd Journal of Internal Medicine 250: 37-42 
right kidney was found to be hypertrophic. Radionuclide imaging confirmed these findings (Fig. 2). Laboratory tests indicated normal renal function.

\section{Patient $V-2$}

The second son of patient IV-1. He had displayed persistent constipation with abdominal distension immediately after birth. A barium enema had shown evidence of long-segment megacolon. Following surgical resection, histological examination revealed total aganglionosis and the diagnosis of Hirschsprung's disease was made. At that time the presence of MTC amongst family members was not known.

Subsequently, serum calcitonin levels were found to be normal, in the basal state as well as following pentagastrin stimulation. The availability of DNA analysis made it possible to detect the C620S RET mutation [11] and the patient underwent prophylactic total thyroidectomy. Histopathologically, MTC was diagnosed.

Abdominal ultrasonography was recently performed and left-side renal absence diagnosed. Intravenous pyelography confirmed agenesis of the left kidney, whilst the contralateral kidney displayed compensatory hypertrophy. Renal function was normal.

No renal abnormalities were found in the other family members.

\section{Discussion}

Germline mutations affecting one of five cysteines (Cys 609, 611, 618, 620 and 634) located in the juxtamembrane domain of the RET receptor are responsible for the vast majority of MEN 2A and FMTC cases. These mutations lead to the replacement of a cysteine residue by another amino acid [6].

It has been demonstrated that the oncogenic potential of RET is a consequence of the constitutive tyrosine kinase activity conferred by MEN 2 mutations [12, 13], whilst inactivating RET mutations are believed to be responsible for Hirschsprung's disease and renal agenesis [14]. Therefore, the cosegregation of MEN 2A and Hirschsprung's disease in certain families represents a puzzling phenomenon. The molecular basis of this finding has probably been provided by the experimental work of
Chappuis-Flament et al., who have shown that mutations at cysteines 609, 618 and 620 may result in a marked decrease in the plasma membrane expression of RET protein and, consequently, in the amount of RET covalent dimer [6]. Thus, unique mutations at cysteines 609, 618 and 620 may exert both activating as well as inactivating effects, depending on the tissue in which RET is expressed. Additional mutations in tyrosine kinase coreceptor and/or ligand genes may have a role in this pathological process [15].

During development, the receptor tyrosine kinase encoded by the RET proto-oncogene is expressed in a variety of tissues including the peripheral and central nervous systems and the kidney [16, 17]. In the latter, factors from the metanephric mesenchyme induce the growth and repeated branching of the ureteric bud, which gives rise to the collecting duct system and also induces nephrogenesis [18]. It has been ascertained that the signalling pathways required for this process include the RET ligand GDNF, produced in the metanephric mesenchyme, the receptor tyrosine kinase RET, and the coreceptor GFR $\alpha-1$ (GDNF family receptor $\alpha$ one), both of which are expressed in the ureteric bud $[8,9,19]$. This has clearly been demonstrated in experimental animals: both GDNF and ret null mice display severe renal abnormalities and lack enteric neurones in a pattern that resembles human Hirschsprung's disease $[7,20]$.

It has recently been shown that RET is also expressed in the human foetal kidney and its highest expression coincides with a stage of renal morphogenesis in which the ureteric bud undergoes rapid branching [21]. RET gene expression has also been found in human enteric neuroblasts of the developing enteric nervous system [22]. Yet, despite these findings, only few cases of renal agenesis have been reported in Hirschsprung's disease patients [22] and no case of renal malformation has been described in patients with FMTC.

In the light of available data regarding the role of the RET gene in experimental animals and in humans, the finding of renal agenesis in our two patients with a C620S mutation cannot be considered purely coincidental.

We believe that the association we have observed is not necessarily exceptional. It is possible that renal abnormalities have not been diagnosed in MEN 2 patients because abdominal imaging is not routinely 
performed in such cases, given that the screening for pheochromocytoma and hyperparathyroidism in patients with MTC is generally based on laboratory tests [23].

Therefore, we suggest that an extensive study should be performed in order to establish the actual prevalence of major renal abnormalities in families with MEN 2 and mutations at cysteines 609,618 or 620 . We also propose that noninvasive and cost-effective diagnostic procedures, such as ultrasonography, should be routinely performed, in the setting of the initial clinical evaluation, in patients with suspected MEN, and their relatives, in order to detect possible renal alterations, as renal malformations may have medical implications even for asymptomatic patients. Subjects with unilateral renal absence require lifetime follow-up [24], as hypertension and various degrees of renal insufficiency have been reported in long-term studies of patients with a single kidney $[25,26]$.

Despite the general expectation that mutations in a single gene are associated with one distinct phenotype, recent reports have shown that mutations in the receptor tyrosine kinase gene RET may lead to four different phenotypes (FMTC, MEN 2A, MEN 2B and Hirschsprung's disease) [27]. Our findings seem to indicate the existence of a fifth phenotype associated with constitutional mutations of the RET proto-oncogene.

\section{Acknowledgements}

This paper is dedicated to the memory of Professor Angelo Caniggia.

This paper has been supported by the Italian Ministry of University and Scientific Research (MURST, Programmi di Ricerca di Rilevante Interesse Nazionale, n. 23, 1998).

\section{References}

1 Schimke R. Genetic aspects of multiple endocrine neoplasia. Annu Rev Med 1984; 35: 25-31.

2 Eng C. The RET proto-oncogene in multiple endocrine neoplasia type 2 and Hirschsprung's disease. $N$ Engl J Med 1996; 335: 943-51.

3 Eng C, Klayton D, Schuffenecker I et al. The relationship between specific RET proto-oncogene mutations and disease phenotypes in multiple endocrine neoplasia type 2. JAMA 1996; 276: 1575-9.
4 Takahashi M, Cooper G. RET transforming gene encodes a fusion protein homologous to tyrosine kinases. Mol Cell Biol 1987; 7: 1378-85.

5 Mulligan LM, Marsh DJ, Robinson BG et al. Genotype-phenotype correlation in multiple endocrine neoplasia type 2 : report of the International RET Mutation Consortium. J Intern Med 1995; 238: 343-6.

6 Chappuis-Flament S, Pasini A, Vita GD et al. Dual effect on the RET receptor of MEN 2 mutations affecting specific extracytoplasmic cysteines. Oncogene 1998; 17: 2851-61.

7 Schuchardt A, D'Agati V, Larsson-Blomberg L, Costantini F, Pachnis V. Defects in the kidney and enteric nervous system of mice lacking the tyrosine kinase receptor Ret. Nature 1994; 367: 380-3.

8 Treanor JJ, Goodman L, de Sauvage F et al. Characterization of a multicomponent receptor for GDNF. Nature 1996; 382: $80-3$.

9 Jing S, Wen D, Yu Y et al. GDNF-induced activation of the ret protein tyrosine kinase is mediated by GDNFR-alpha, a novel receptor for GDNF. Cell 1996; 85: 1113-24.

10 Loré F, Di Cairano G, Talidis F. Unilateral renal agenesis in a family with medullary thyroid carcinoma. N Eng J Med 2000; 342: 1218-9.

11 Romeo G, Ceccherini I, Celli J et al. Association of multiple endocrine neoplasia type 2 and Hirschsprung disease. J Intern Med 1998; 243: 515-20.

12 Asai N, Iwashita T, Matsuyama M, Takahashi M. Mechanism of activation of the ret proto-oncogene by multiple endocrine neoplasia 2A mutations. Mol Cell Biol 1995; 15: 1613-9.

13 Santoro M, Carlomagno F, Romano A et al. Activation of RET as a dominant transforming gene by germline mutations of MEN2A and MEN2B. Science 1995; 267: 381-3.

14 Reynolds LF, Eng C. RET mutations in multiple endocrine neoplasia type 2 and Hirschsprung disease. Curr Opin Pediatr 1995; 7: 702-9.

15 Hansford JR, Mulligan LM. Multiple endocrine neoplasia type 2 and RET: from neoplasia to neurogenesis. J Med Genet 2000; 37: $817-27$.

16 Pachnis V, Mankoo B, Costantini F. Expression of the c-ret proto-oncogene during mouse embryogenesis. Development 1993; 119: 1005-17.

17 Tsuzuki T, Takahashi M, Asai N, Iwashita T, Matsuiama M, Asai J. Spatial and temporal expression of the ret protooncogene product in embryonic, infant and adult rat tissues. Oncogene 1995; 10: 191-8.

18 Bard J, McConnell J, Davies J. Towards a genetic basis for kidney development. Mech Dev 1994; 48: 3-11.

19 Srinivas S, Wu Z, Chen C, D'Agati V, Costantini F. Dominant effects of RET receptor misexpression and ligand-independent RET signaling on ureteric bud development. Development 1999; 126: 1375-86.

20 Sanchez M, Silos-Santiago I, Frisen J, He B, Lira S, Barbacid M. Renal agenesis and the absence of enteric neurons in mice lacking GDNF. Nature 1996; 382: 70-3.

21 Ivanchuk S, Eng C, Cavenee W, Mulligan L. The expression of RET and its multiple splice forms in developing human kidney. Oncogene 1997; 14: 1811-8.

22 Attié-Bitach T, Abitbol M, Gérard M et al. Expression of the RET proto-oncogene in human embryos. Am J Med Genet 1998; 80: 481-6.

23 Eng C. RET proto-oncogene in the development of human cancer. J Clin Oncol 1999; 17: 380-93. 
24 Robson WL, Leung AK, Rogers RC. Unilateral renal agenesis. Adv Pediatr 1995; 42: 575-92.

25 Argueso LR, Ritchey ML, Boyle ET, Milliner DS, Bergstralh EJ, Kramer SA. Prognosis of patients with unilateral renal agenesis. Pediatr Nephrol 1992; 6: 412-6.

26 Wikstad I, Celsi G, Larsson L, Herin P, Aperia A. Kidney function in adults born with unilateral renal agenesis or nephrectomized in childhood. Pediatr Nephrol 1988; 2: 177-82.

27 van Heynengen V. One gene - four syndromes. Nature 1994; 367: 319-20.
Received 27 December 2000; revision received 20 March 2001; accepted 20 March 2001.

Correspondence: Fausto Loré MD, Endocrinology Unit, University of Siena, Policlinico Le Scotte, 53100 Siena, Italy (fax: +390577 586187; e-mail: lore@unisi.it). 